Vi brati on anal ysi s of a ci rcul ar di sk tensi oned by rol Iing using fi ni te el ement met hod

\begin{tabular}{|l|l|}
\hline 著者 & KURATAN F., YANO S. \\
\hline $\begin{array}{l}\text { j our nal or } \\
\text { publ i cat i on t i t l e }\end{array}$ & Ar chi ve of Appl i ed Mechani cs \\
\hline vol une & 70 \\
\hline page range & $279-288$ \\
\hline year & $2000-05$ \\
\hline URL & ht t p: //hdl . handl e. net /10098/5250 \\
\hline
\end{tabular}




\title{
Vibration analysis of a circular disk tensioned by rolling using finite element method
}

\author{
F. Kuratani, S. Yano
}

Summary The paper proposes a method in finite element analysis for estimating natural frequencies of a disk tensioned by rolling, without the use of eigenvalue analysis. The natural frequencies of a disk vary when the localized plastic deformation caused by roll-tensioning induces residual stresses. Tensioning is used for improving the dynamic stability of circular saws; the optimal condition of rolling can be predicted from natural frequency characteristics. In the proposed method, the natural frequencies after rolling are easily estimated from the mode shapes of the disk before rolling and the stress distribution after rolling. The method is based on ideas similar to thermal stress and sensitivity analysis rather than on eigenvalue analysis. The effectiveness of the method is shown by comparing the natural frequency characteristics obtained by this method with those by eigenvalue analysis.

Key words vibration, roll-tensioning, plastic deformation, eigenfrequency, FEM

1

Introduction

Circular saws are widely used for cutting and forming wood. Since large transverse displacements by resonance occasionally occur in cutting works, a process called "tensioning has been traditionally used for improving the dynamic stability. It is reported in $[1,2]$ that the resonance is caused by the reduction of natural frequencies of the sawblade due to the thermal compressive stress induced by cutting heat in the peripheral region. It is desired, therefore, that the tensile stress should be previously induced by tensioning, so that the natural frequency concerning critical speed instability would be enhanced. Especially, the process using localized plastic deformation by rollers, called roll-tensioning, and the automation of this process are expected to improve the performance similarly to the skill and experience of craftsmen.

Some studies on roll-tensioning have been done in [3], where residual stresses of disks induced by roll-tensioning were analyzed and compared with experimental results. In $[4,5]$ natural frequencies of a disk tensioned by rollers as well as residual stresses were obtained. These studies contained theoretical analyses for uniform thin disks, however, in practical sawblades, there are some holes against heat, slots and tips at the periphery. It is not easy to develop analytical theories for such sawblades. Contrary to this, sawblades with slots and tips can be easily modeled within the FEM (Finite Element Method). In order to determine the optimal rolling condition, it is important to grasp their relation to natural frequency characteristics. The use of eigenvalue analysis for that purpose is accompanied by much computational effort.

In this paper, we propose a method using FEM for easy estimates of natural frequencies of disks tensioned by rolling, which is based on an idea similar to the sensitivity analysis. The

Received 18 June 1998; accepted for publication 8 April 1999

F. Kuratani

Hyogo University of Teacher Education

Simokume 942-1

673-1494 Yashiro-cho, Kato-gun

Hyogo, Japan

S. Yano

Faculty of Human Development

Kobe University

Tsurukabuto 3-11, Nada-ku

657-8501 Kobe-shi, Japan 
variation in stiffness due to rolling is analyzed in a way of temperature loading as shown in [3], instead of the static stress analysis. Natural frequency characteristics for various rolling and clamping positions by a flange are obtained by the proposed method, and it is shown that there exists an optimal position of rolling. The effectiveness of the proposed method is ascertained by comparison with results obtained by eigenvalue analysis. The role of tensioning is assessed from the change in natural frequencies and mode shapes.

\section{2}

\section{Tensioning of circular sawblades by rollers}

In the tensioning process, a sawblade is compressed within a certain annular contact zone between two opposing rollers as shown in Fig. 1. The sawblade is plastically deformed in the rolling region as shown in Fig. 2, [3], and there occur tensile residual stresses in the inner and outer regions. Especially at the outer edge, the tensile residual stresses become large. The tensile stresses overcome thermal compressive stresses induced during cutting, and result in enhancing natural frequencies. Generally, it is known that the critical speed instability in rotating disks occurs when the backward-traveling wave frequency is equal to zero, $[3,4]$. In the case of circular saws, the thermal stresses also affect the stability, and it is necessary to enhance natural frequencies by tensioning.

As reported in [6], vibration characteristics are improved or not improved according to the stress distribution within the sawblade induced by rolling, even if there occur residual tensile stresses at the periphery. The residual stress distribution is affected by roller path position, roller load, number of rolling processes and so on. The roller path position greatly affects the residual stress distribution, and it is an important problem to determine the optimal rolling position, [7]. However, it is difficult to determine the optimal position from stress analysis alone. We investigate the optimal rolling position by vibration analysis, using FEM and a method for estimating the change in natural frequencies.

\section{3}

\section{Analysis of the problem}

We explain the method using FEM for analyzing and estimating vibration characteristics of thin circular disks tensioned by rolling.

\section{1}

\section{Equation of motion}

In general, the equation of motion of a disk modeled by FEM is described by

$$
[M]\{\ddot{u}\}+[K]\{u\}=\{f\},
$$
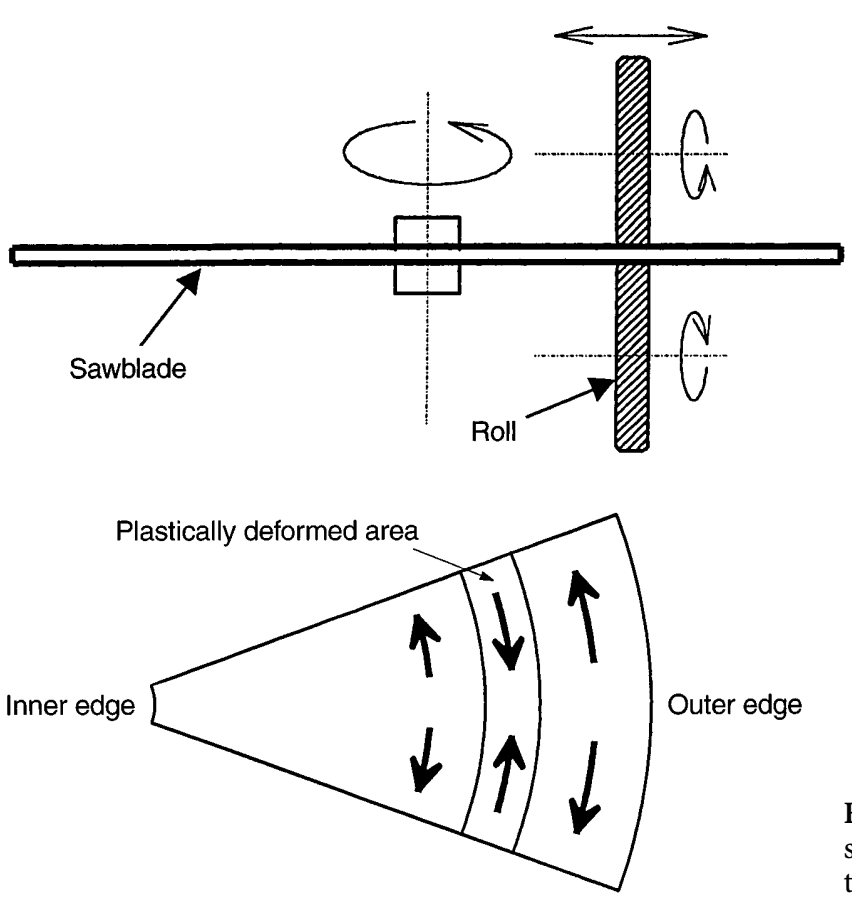

Fig. 1. Roll tensioning of a circular sawblade
Fig. 2. Typical distribution of residual stresses induced by the plastic deformation at rolling 
where $[M]$ and $[K]$ are mass and stiffness matrices; $\{u\}$ and $\{f\}$ are deflection and external force vectors, respectively. Natural frequencies of disks can be changed by roll-tensioning, which induces a certain pattern of residual stresses by the localized plastic deformation. The mass distribution is not changed due to rolling, but the stiffness characteristics vary greatly. Let the variation in stiffness from $[K]$ be $\left[K^{(\sigma)}\right]$. Then, the equation of motion of a disk after rolling is described by

$[M]\{\ddot{\tilde{u}}\}+\left([K]+\left[K^{(\sigma)}\right]\right)\{\tilde{u}\}=\{f\}$,

where $\{\tilde{u}\}$ denotes the deflection of a disk after rolling. In the case of shell elements and plane stress, the elements of the matrix $\left[K^{(\sigma)}\right]$ are given by

$\left[K_{i}^{(\sigma)}\right]=\int_{V}\left[B_{d}\right]^{T}\left[\begin{array}{cccccc}\sigma_{x} & & & & & \\ \tau_{x y} & \sigma_{y} & & & \text { sym. } \\ 0 & 0 & \sigma_{x} & & & \\ 0 & 0 & \tau_{x y} & \sigma_{y} & & \\ 0 & 0 & 0 & 0 & \sigma_{x} & \\ 0 & 0 & 0 & 0 & \tau_{x y} & \sigma_{y}\end{array}\right]\left[B_{d}\right] \mathrm{d} V$,

where $\sigma_{x}$ and $\sigma_{y}$ are normal stresses in the $x$ and $y$ directions in the element coordinates and $\tau_{x y}$ is the shearing stress; $\left[B_{d}\right]$ is a matrix whose components consist of derivatives of deflection. The symbol $T$ means the transpose of a matrix, and $V$ is the volume.

\section{2}

\section{Stress analysis for $\left[K^{(\sigma)}\right]$}

In order to calculate $\left[K^{(\sigma)}\right]$, we must know the stress distribution of a disk after rolling. For obtaining the stress distribution, we adopt a similar thermal stress analysis as in Ref. [3], without directly analyzing plastic deformation by rolling. Namely, a temperature distribution load, which is considered to induce the same stress distribution as that by plastic deformation, is used for the analysis. It is assumed that the plastic deformation induced by rolling has the maximum value at the center of rolling region and decreases toward the peripheral region rapidly and continuously. We use the temperature distribution of the Gaussian function type

$T(r)=A \exp \left\{-\frac{\left(r-r_{c}\right)^{2}}{2 s^{2}}\right\}$,

where $T(r)$ is the temperature at the radius $r, A$ is the maximum temperature, $r_{c}$ is the radius at the center of the temperature distribution, and $s$ is the standard deviation related to the width of the distribution determined from the roller load, the number and the width of rolling. The distribution of $95 \%$ is included within the extent of $4 s$ that is considered to be an appropriate rolling width, [3].

This analytical method has the advantage of less computational effort than a method directly analyzing the plastic deformation. The effectiveness of this method is shown by the comparison of both analytical results and measurements concerning residual stresses,[3], and natural frequencies, [4], of tensioned disks.

\section{3}

\section{Vibration analysis of the variation in natural frequencies}

Natural frequencies of a disk with a stress distribution can be obtained from the following eigenvalue problem, after calculating $\left[K^{(\sigma)}\right]$ by the thermal stress analysis,

$$
\left([K]+\left[K^{(\sigma)}\right]-\tilde{\omega}^{2}[M]\right)\{\tilde{\phi}\}=\{0\},
$$

where $\tilde{\omega}$ and $\{\tilde{\phi}\}$ with the upper tilde $\sim$ denote the natural frequency and the mode shape vector of a disk after rolling, respectively. To obtain natural frequency curves for the center radius $r_{c}$, it is necessary to determine the optimal rolling radius. A large amount of eigenvalue analysis is required for that. 
We propose, therefore, a method for easy prediction of natural frequencies after rolling similar to the sensitivity analysis rather than the eigenvalue analysis. Let $\omega_{i}$ and $\left\{\phi_{i}\right\}$ be the $i$-th natural angular frequency and the mode shape vector of a disk before rolling, respectively. These satisfy the following relation:

$\left([K]-\omega_{i}^{2}[M]\right)\left\{\phi_{i}\right\}=\{0\}$.

Considering $r_{c}$ as a design variable, the partial derivative of Eq. (6) with respect to $r_{c}$ produces

$\left([K]-\omega_{i}^{2}[M]\right) \frac{\partial\left\{\phi_{i}\right\}}{\partial r_{c}}+\left(\frac{\partial[K]}{\partial r_{c}}-\omega_{i}^{2} \frac{\partial[M]}{\partial r_{c}}\right)\left\{\phi_{i}\right\}-\frac{\partial \omega_{i}^{2}}{\partial r_{c}}[M]\left\{\phi_{i}\right\}=\{0\}$.

Multiplying Eq. (7) by $\left\{\phi_{i}\right\}^{T}$ from the left side, and considering the symmetry of $[M]$ and $[K]$, we obtain the sensitivity of the eigenvalues (squared frequencies) for $r_{c}$

$\frac{\partial \omega_{i}^{2}}{\partial r_{c}}=\frac{\left\{\phi_{i}\right\}^{T}\left(\frac{\partial[K]}{\partial r_{c}}-\omega_{i}^{2} \frac{\partial[M]}{\partial r_{c}}\right)\left\{\phi_{i}\right\}}{\left\{\phi_{i}\right\}^{T}[M]\left\{\phi_{i}\right\}}$.

Since for a disk after rolling the matrix $[M]$ is not changed, but $[K]$ is changed by $\left[K^{(\sigma)}\right]$, the variation in the $i$-th eigenvalue for arbitrary $r_{c}$ is given by

$\Delta \omega_{i}^{2}\left(r_{c}\right)=\frac{\left\{\phi_{i}\right\}^{T}\left[K^{(\sigma)}\right]\left\{\phi_{i}\right\}}{\left\{\phi_{i}\right\}^{T}[M]\left\{\phi_{i}\right\}}$.

Equation (9) means that once the mode vector before rolling $\left\{\phi_{i}\right\}$ is obtained, natural frequencies after rolling can be easily predicted without eigenvalue analysis.

Equation (9) can also be derived in another way. We assume that the mode vector after rolling $\{\tilde{\phi}\}$ is described by superposition of mode vectors before rolling as

$\{\tilde{\phi}\}=[\Phi]\{\xi\}=\left[\left\{\phi_{1}\right\} \cdots\left\{\phi_{n}\right\}\right]\{\xi\}$,

where $\{\xi\}$ is the modal coordinate vector. Substituting Eq. (10) into Eq. (5) and multiplying the resulting equation by $[\Phi]^{T}$ from the left side, we obtain

$$
\begin{aligned}
& \left(\lceil k\rfloor+[\Phi]^{T}\left[K^{(\sigma)}\right][\Phi]-\tilde{\omega}^{2}\lceil m\rfloor\right)\{\xi\}=\{0\} ; \\
& \lceil m\rfloor=[\Phi]^{T}[M][\Phi], \quad\lceil k\rfloor=[\Phi]^{T}[K][\Phi] .
\end{aligned}
$$

Here $\lceil m\rfloor$ and $\lceil k\rfloor$ are diagonal matrices whose elements consist of modal masses $m_{i}(i=1, \ldots, n)$ and modal stiffnesses $k_{i}$, respectively. If $[\Phi]^{T}\left[K^{(\sigma)}\right][\Phi]$ also becomes a diagonal matrix, the lefthand side of Eq. (11) becomes a system of uncoupled equations, whose $i$-th equation is expressed by

$\left(k_{i}+\left\{\phi_{i}\right\}^{T}\left[K^{(\sigma)}\right]\left\{\phi_{i}\right\}-\tilde{\omega}_{i}^{2} m_{i}\right) \xi_{i}=0$.

Then, looking for natural frequencies after rolling we obtain

$\tilde{\omega}_{i}^{2}=\omega_{i}^{2}+\frac{\left\{\phi_{i}\right\}^{T}\left[K^{(\sigma)}\right]\left\{\phi_{i}\right\}}{m_{i}}$.

It is found from $m_{i}=\left\{\phi_{i}\right\}^{T}[M]\left\{\phi_{i}\right\}$ that the second term in the right-hand side of Eq. (13), which means the variation from $\omega_{i}^{2}\left(=k_{i} / m_{i}\right)$ before rolling, is equal to Eq. (9) and the natural frequency characteristic after rolling can be predicted from Eq. (9).

Next, we consider the prediction accuracy of Eq. (9) or (13) in relation to diagonal $[\Phi]^{T}\left[K^{(\sigma)}\right][\Phi]$. Let $[\Phi]$ and $[\tilde{\Phi}]$ be the modal matrix before and after rolling, respectively. The matrix $[\Phi]^{T}\left[K^{(\sigma)}\right][\Phi]$ becomes a diagonal matrix when vibration modes before and after rolling are not changed regardless of stress distribution, that it, $[\tilde{\Phi}]=[\Phi]$ holds. This condition is introduced as follows: since vibration modes after rolling have orthogonality with respect to the stiffness matrix $[K]+\left[K^{(\sigma)}\right]$, the relation 
$[\tilde{\Phi}]^{T}\left([K]+\left[K^{(\sigma)}\right]\right)[\tilde{\Phi}]=\lceil\tilde{k}\rfloor$,

holds. If $[\tilde{\Phi}]=[\Phi]$ holds, the following relation is also satisfied:

$\lceil k\rfloor+[\Phi]^{T}\left[K^{(\sigma)}\right][\Phi]=\lceil\tilde{k}\rfloor$,

Since $\lceil k\rfloor$ and $\lceil\tilde{k}\rfloor$ are diagonal matrices, $[\Phi]^{T}\left[K^{(\sigma)}\right][\Phi]$ must also be diagonal under the condition that $[\tilde{\Phi}]=[\Phi]$. Therefore, it is expected that the prediction of natural frequencies by Eq. (9) has high accuracy when there is little difference between mode shapes before and after rolling, because then $[\Phi]^{T}\left[K^{(\sigma)}\right][\Phi]$ is approximately diagonal. Later we calculate and ascertain whether or not

$\left[\hat{K}^{(\sigma)}\right]=[\Phi]^{T}\left[K^{(\sigma)}\right][\Phi]$

is approximately a diagonal matrix. Concerning the condition that $[\tilde{\Phi}]=[\Phi]$, we ascertain by checking the orthogonality of the following matrix with respect to mass matrix:

$[\hat{m}]=[\tilde{\Phi}]^{T}[M][\Phi]$.

When $[\Phi]$ and $[\tilde{\Phi}]$ are normalized so that modal masses become unity, $[\hat{m}]$ will be approximately a unit matrix.

4

\section{Numerical examples and considerations}

First, we obtain the variation in natural frequencies for various $r_{c}$ by eigenvalue analysis and show that there exists an optimal rolling radius. Next, we compare the variation in natural frequencies by Eq. (9) with that by eigenvalue analysis. A sawblade is modeled by a circular disk of the outer diameter $255 \mathrm{~mm}$, as shown in Fig. 3. The disk is analyzed by FEM (the software ANSYS) using four-node plane shell elements.

\section{1}

\section{Stress distribution}

We consider the case where rolling is performed around the center radius $65 \mathrm{~mm}$. Figure 4 shows the temperature distribution $T(r)$ which is assumed to induce an equivalent stress distribution to that by rolling. Parameters of Eq. (4) are $r_{c}=65 \mathrm{~mm}, A=50^{\circ} \mathrm{C}$ and $s=5$.

Figure 5 shows a stress distribution within the disk induced by temperature distribution loading shown in Fig. 4, where only 1/12 part of the disk is drawn. It is found that large circumferential compressive stresses are recognized within the region of $T(r)$ loading, and there appear circumferential tensile stresses.

\section{2}

\section{Natural frequency characteristics by eigenvalue analysis}

We investigate the effect $r_{c}$ in $T(r)$ on natural frequencies after rolling $\tilde{\omega}_{i}$. Even if the temperature loading is the same as in Fig. $4, \tilde{\omega}_{i}$ varies with $r_{c}$ and there exists the optimal rolling position for enhancing $\tilde{\omega}_{i}$. Since a practical circular sawblade is clamped by a flange, the clamping radius $r_{f}$ affects the optimal rolling position, and then $\tilde{\omega}_{i}$ curves are calculated for $r_{c}$ and $r_{f}$. The disk for analysis is assumed to be perfectly fixed in the region of $r \leq r_{f}$. The outer edge is free. Regarding the relationship between tensioning and the number of nodal diameters of vibration modes $N_{d}$, it is reported in [6] that compressive stresses during cutting make natural frequencies of $N_{d}=0$ and 1 increase, but those of $N_{d} \geq 2$ decrease. Therefore, it becomes necessary to enhance natural frequencies of $N_{d} \geq 2$ beforehand, and appropriate positions for rolling should be selected so that natural frequencies of $N_{d} \geq 2$ become large.

Figure 6 shows $\tilde{\omega}_{i}$ curves of $N_{d}=0$ to 4 calculated by FEM when $r_{c}$ is varied from $25 \mathrm{~mm}$ to $105 \mathrm{~mm}$ by $10 \mathrm{~mm}$, with keeping the temperature distribution in Fig. 4. Figure 6a-c present the results for $r_{f}=25 \mathrm{~mm}, 55 \mathrm{~mm}$ and $75 \mathrm{~mm}$, respectively. For reference, natural frequencies before rolling $\omega_{i}$ are marked at $r_{c}=0$. It is seen that the tendency of curves of $N_{d}=2$ to 4 differs from that of $N_{d}=0$ and 1. For example, in Fig. 6a, natural frequencies of $N_{d}=2$ to 4 increase with $r_{c}$ until they reach the maximum values near $r_{c}=65 \mathrm{~mm}$; then they decrease. They become smaller than $\omega_{i}$ for $r_{c}$ near the outer edge, and the effect of rolling is not 


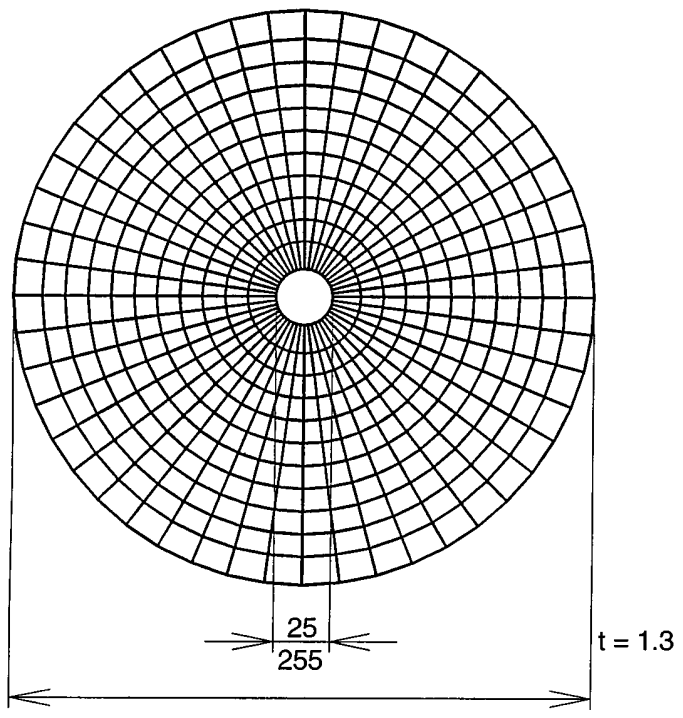

Fig. 3. FEM model for the disk
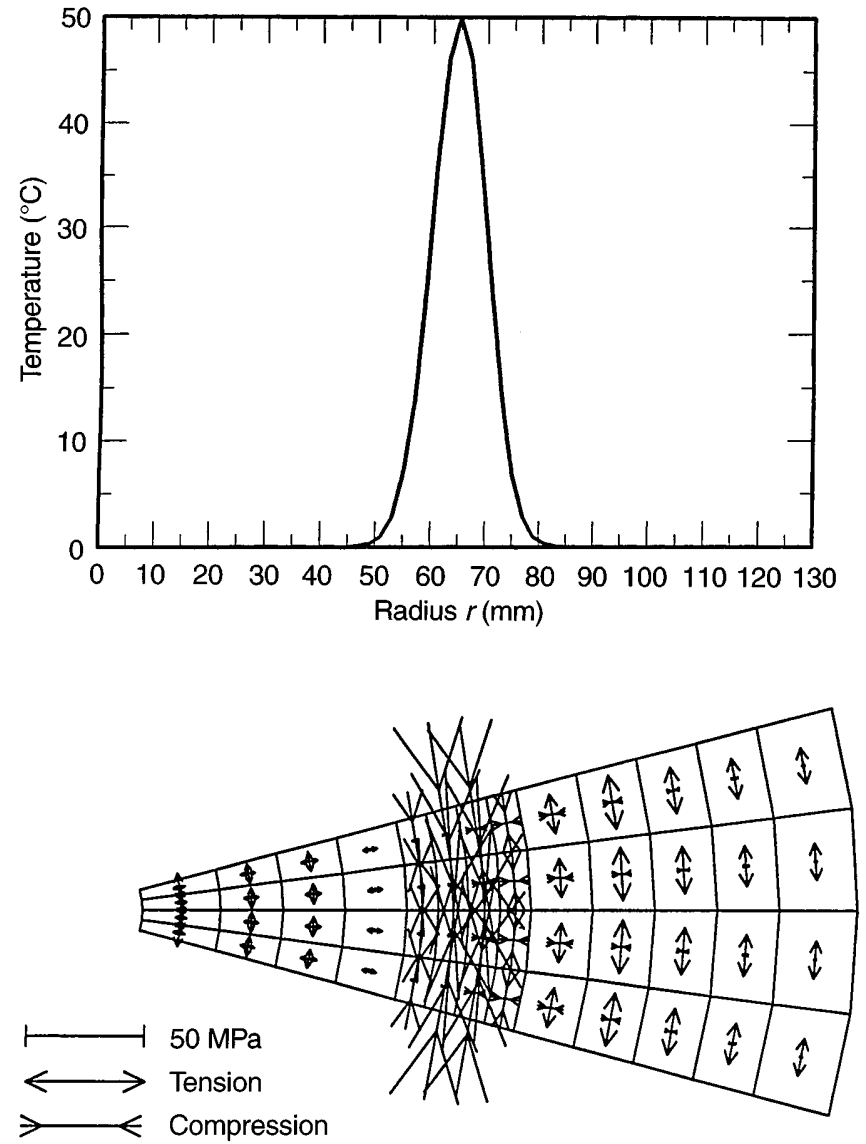

Fig. 4. Temperature distribution assumed for residual stresses induced by rolling

Fig. 5. Stress distribution in the disk subjected to the temperature distribution shown in Fig. 4

recognized. Contrary to this, natural frequencies of $N_{d}=0$ and 1 reach the minimum values near $r_{c}=45 \mathrm{~mm}$; then they increase. In Fig. $6 \mathrm{~b}$ and c, curves of $N_{d}=2$ are not much changed for $r_{c}$. Comparing Fig. 6a-c, such $r_{c}$, for which $\tilde{\omega}_{i}$ of $N_{d}=3$ and 4 becomes maximal, also increases for larger $r_{f}$. It is found that the optimal rolling position should be determined from $\tilde{\omega}_{i}$ curves for $r_{c}$ and $r_{f}$.

Next, we consider the optimal rolling position from the viewpoint of sensitivity of natural frequencies in relation to the difference of tendency between curves of $N_{d}=3$ and 4 and curves of $N_{d}=0$ and 1 . The reason why natural frequencies are varied due to rolling is that stiffness characteristics within the disk are locally changed by the stress distribution. In order to investigate the effect of stiffness characteristics on the change in natural frequencies, a disk is 

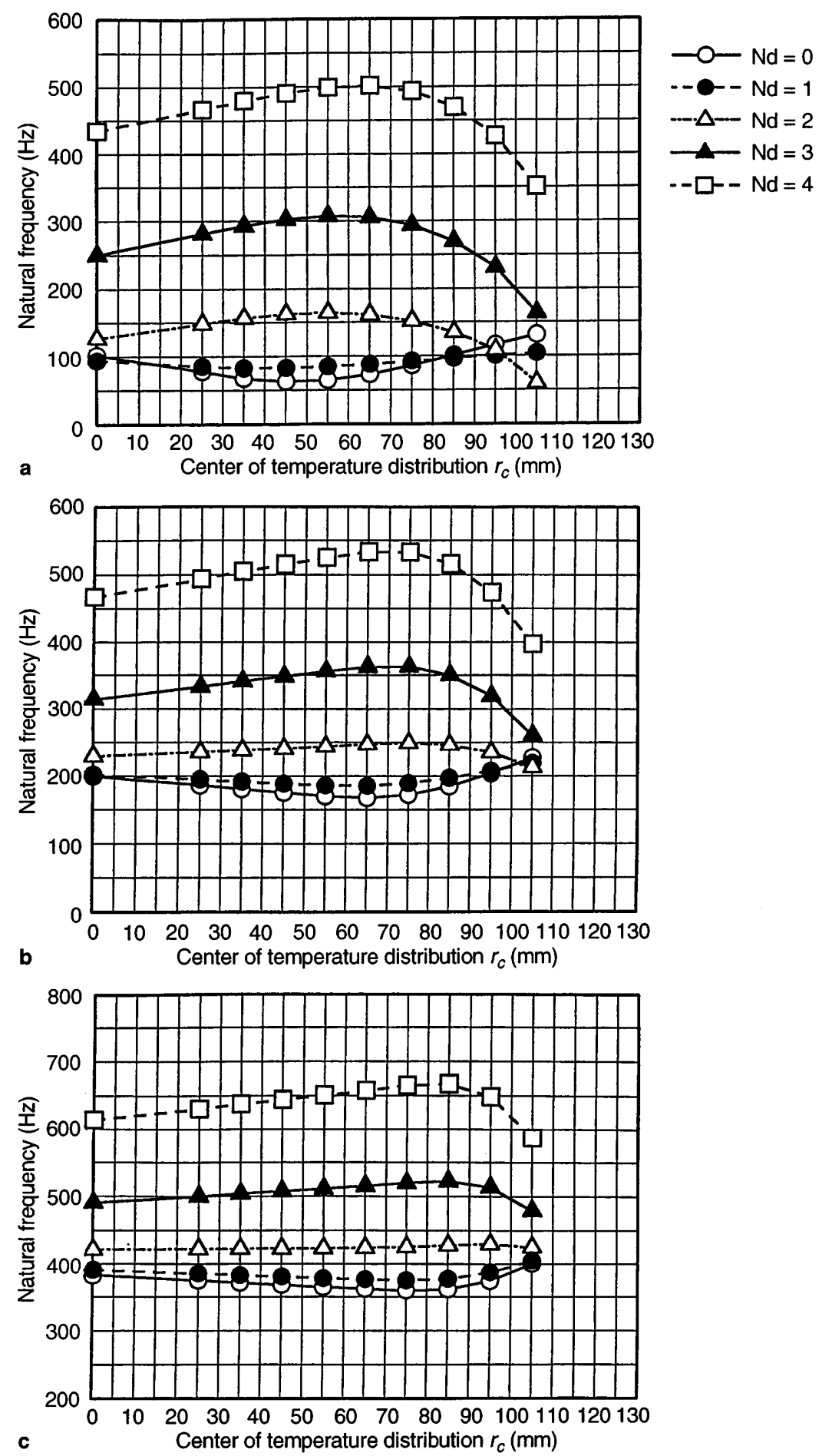

Fig. 6a-c. Relation between natural frequencies and $r_{c}$. a $r_{f}=25 \mathrm{~mm}, \mathbf{b} r_{f}=55 \mathrm{~mm}, \mathbf{c} r_{f}=75 \mathrm{~mm}$

analyzed whose stiffness for the annular zone of the width $10 \mathrm{~mm}$ centered at the radius $r_{a}$ is enhanced by $1 \%$. The relationship between the sensitivity of natural frequencies and $r_{a}$ is shown in Fig. 7 for $r_{f}=25 \mathrm{~mm}$. The sensitivity for $N_{d}=0$ and 1 monotonously decreases toward the outer edge, whereas the sensitivity for $N_{d}=3$ and 4 substantially increases. Remember the results by $T(r)$ loading as shown in Fig. 5. Large compressive stresses occur near $r_{c}$, and tensile stresses occur in the inner and outer regions, where the absolute values of compressive stresses are quite larger than tensile stresses. When $r_{c}$ is selected to be near the outer edge, natural frequencies of $N_{d}=3$ and 4 decrease due to the compressive stresses as shown in Fig. 6a because the sensitivity for $N_{d}=3$ and 4 is high. 


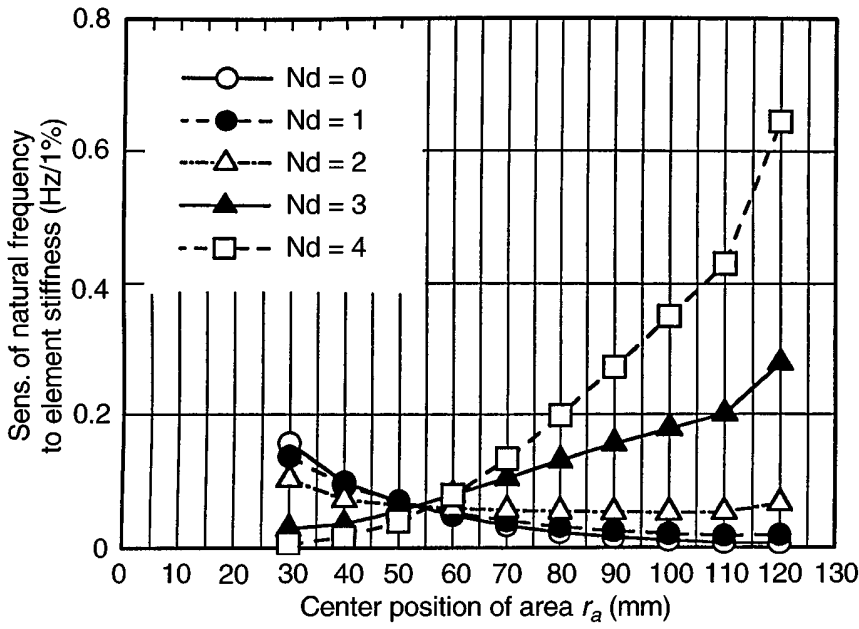

4.3

Verification of estimation of natural frequencies by Eq. (9)

Figure 8 shows values of $\Delta \omega_{i}=\tilde{\omega}_{i}-\omega_{i}$ estimated by Eq. (9) for $r_{c}=25 \mathrm{~mm}$ to $105 \mathrm{~mm}$ by $10 \mathrm{~mm}$. Figures $8 \mathrm{a}-\mathrm{c}$ present the results for $r_{f}=25 \mathrm{~mm}, 55 \mathrm{~mm}$ and $75 \mathrm{~mm}$, respectively, which correspond to Fig. $6 \mathrm{a}-\mathrm{c}$ by eigenvalue analysis. Comparing them we observe, that natural frequencies of $N_{d}=2$ to 4 become maximal for such values of $r_{c}$ which well coincide with each other except for $N_{d}=2$ in Fig. 8c. We compare values for $N_{d}=4$ for $r_{f}=25 \mathrm{~mm}$, at which large variation takes place. In Fig. $8 \mathrm{a}, \Delta \omega=70.0 \mathrm{~Hz}$ at $r_{c}=65 \mathrm{~mm}$, whereas in Fig. 6a $\Delta \omega=67.6 \mathrm{~Hz}$ from $\tilde{\omega}=502.1 \mathrm{~Hz}$ and $\omega=434.5 \mathrm{~Hz}$. The difference between both values is $2.4 \mathrm{~Hz}$ and small enough. Accordingly, it is possible to predict the variation in natural frequencies by Eq. (9) with high accuracy.

Next, we check two matrices of Eqs. (16) and (17) concerning the accuracy of Eq. (9). Table 1 shows the components of $\left[\hat{K}^{(\sigma)}\right]$ with respect to $N_{d}=0$ to 4 for $r_{c}=65 \mathrm{~mm}$ and $r_{f}=25 \mathrm{~mm}$. Most of nondiagonal components are zero. There appear nonzero components between $N_{d}=0$ and 3 and between $N_{d}=1$ and 4 , but these values are quite smaller than the diagonal components. Therefore, $\left[\hat{K}^{(\sigma)}\right]$ is approximately diagonal, and it is considered that Eq. (9) has a high accuracy. Table 2 shows components of $[\hat{m}]$ in Eq. (17) for $r_{f}=25 \mathrm{~mm}$ and $r_{c}=65 \mathrm{~mm}$. All the nondiagonal components are zero, and the diagonal ones, except for $N_{d}=3$, become unity. Since $[\hat{m}]$ is approximately a unit matrix, the condition that $[\tilde{\Phi}]=[\Phi]$ also holds. Accordingly, it is concluded that natural frequencies are greatly varied due to rolltensioning, but the mode shapes change little.

5

\section{Conclusions}

A method in finite element analysis for estimating natural frequencies of disks tensioned by rolling without the use of eigenvalue analysis has been proposed. Instead of static stress analysis, the temperature distribution load assumed for plastic deformation was used for calculating the variation in stiffness due to rolling. The summary of results is as follows:

(1) It is ascertained from verification by Eqs. (16) and (17) and comparison with results by eigenvalue analysis that the change in natural frequencies can be predicted from Eq. (9)

Table 1. Matrix $\left[\hat{K}^{(\sigma)}\right]$ for $r_{c}=65 \mathrm{~mm}$ and $r_{f}=25 \mathrm{~mm}$

\begin{tabular}{llllll}
\hline $\mathrm{Nd}$ & 0 & 1 & 2 & 3 & 4 \\
\hline 0 & $-1.70 \times 10^{5}$ & 0.00 & 0.00 & 0.00 & -6.26 \\
1 & 0.00 & $-3.41 \times 10^{4}$ & 0.00 & -4.35 & 0.00 \\
2 & 0.00 & 0.00 & $3.82 \times 10^{5}$ & 0.00 & 0.00 \\
3 & 0.00 & -4.35 & 0.00 & $1.16 \times 10^{6}$ & 0.00 \\
4 & -6.26 & 0.00 & 0.00 & 0.00 & $2.40 \times 10^{6}$ \\
\hline
\end{tabular}



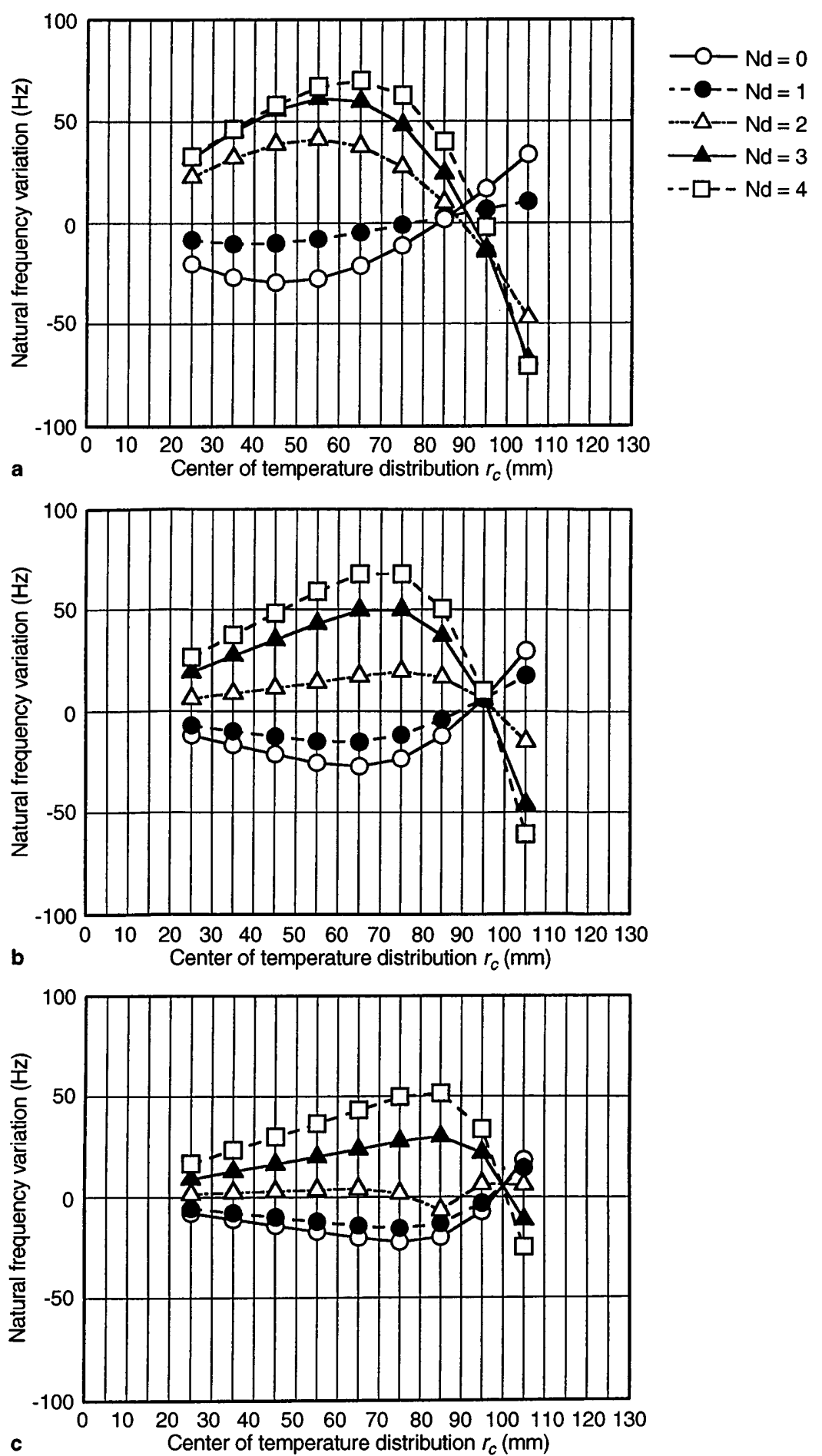

Fig. 8a-c. Variation in natural frequencies for $r_{c}$ by Eq. (9). a $r_{f}=25 \mathrm{~mm}, \mathbf{b} r_{f}=55 \mathrm{~mm}, \mathbf{c} r_{f}=75 \mathrm{~mm}$

Table 2. Matrix $[\hat{m}]$ for $r_{c}=65 \mathrm{~mm}$ and $r_{f}=25 \mathrm{~mm}$

\begin{tabular}{|c|c|c|c|c|c|c|}
\hline $\begin{array}{l}\text { mode shape } \\
\text { after rolling }\end{array}$ & $\mathrm{Nd}$ & 0 & 1 & 2 & 3 & 4 \\
\hline & 0 & 1.00 & 0.00 & 0.00 & 0.00 & 0.00 \\
\hline & 1 & 0.00 & 1.00 & 0.00 & 0.00 & 0.00 \\
\hline & 2 & 0.00 & 0.00 & 1.00 & 0.00 & 0.00 \\
\hline & 3 & 0.00 & 0.00 & 0.00 & 0.93 & 0.00 \\
\hline & 4 & 0.00 & 0.00 & 0.00 & 0.00 & 1.00 \\
\hline
\end{tabular}


with the advantage of less computational effort and high accuracy. It is found that natural frequencies are greatly varied due to rolling, but the mode shapes change little.

(2) For certain rolling radii $r_{c}$ and clamping radii $r_{f}$, natural frequencies of $N_{d} \geq 2$ become smaller than those before rolling, and the purpose of tensioning cannot be achieved. Therefore, it is necessary to determine the optimal rolling position from natural frequency characteristics calculated for various $r_{c}$ and $r_{f}$.

\section{References}

1. Yokochi, H.; Kimura, S.: Thermal buckling of circular saws I (Thermal buckling behaviour of a dishshaped thin circular saw) (in Japanese), J. Jpn. Wood Res. Soc. 37-5 (1991) 415-423

2. Iwata, H.; Yoshida, Y.: Basic study of tensioning effect on thermally stressed disk vibration (in Japanese), Trans. Jpn. Soc. Mech. Eng. 59-563,C (1993) 2015-2019

3. Kimura, S.; Ando, M.: Studies on tensioning of circular saw by rolling pressure, 1 (in Japanese), J. Jpn. Wood Res. Soc. 20-5 (1974) 196-204

4. Schajer, G. S.; Mote, C. D.: Analysis of optimal roll tensioning for circular saw stability, Wood and Fiber Sci. 16-3 (1984) 323-338

5. Iwata, H.; Yoshida, Y.: A basic study of tensioning effect on rotating disk vibration (in Japanese), Trans. Jpn. Soc. Mech. Eng. 58-547,C (1992) 684-689

6. Kuratani, F.; Okita, K.; Yano, S; Iwatsubo, T.: Evaluation of tensioning of circular saw based on the vibration properties (in Japanese), Prepr. Jpn. Soc. Mech. Eng. No. 96-5, IB (1996-8) 475-478

7. Kimura, S.; Asano, I.: Studies on tensioning of circular saw by rolling pressure, 4 (in Japanese), J. Jpn. Wood Res. Soc. 22-7 (1976) 387-392

8. Washizu, K.: Handbook of Finite Element Method - Application (in Japanese), (1983) Baifukan, 128 